\title{
Chapter 14 \\ Parallel Measurement of Ambient and Individual External Radiation in Iitate Village, Fukushima
}

\author{
Yoichi Tao, Muneo Kanno, Soji Obara, Shunichiro Kuriyama, Takaaki Sano, \\ and Katsuhiko Ninomiya
}

\begin{abstract}
This report is based on a survey we conducted in March-June 2017 to measure radiation levels in Iitate Village after the 2011 Fukushima Daiichi nuclear power plant accident. Six NPO members including two evacuees who returned after the evacuation order was lifted in April 2017 took part in the study. Each participant worked in the area each day, carrying an ambient radiation measuring device, two personal radiation measuring devices, and a GPS receiver to record their movement and compare ambient and individual external doses. No matter where they were, results showed that for those taking the same daily route for the same amount of time, the individual dose was about 11 30\% lower than that of the ambient dose; the ratio of ambient to individual external dose was 100 to 70-89. Further measurement and additional data are needed to protect the health of villagers, and to identify areas that require further decontamination.
\end{abstract}

Keywords Fukushima Iitate Village · Fukushima Daiichi Nuclear Accident · Ambient dose $\cdot$ Individual dose $\cdot$ Lifestyle pattern

One Sentence Summary: When measuring individual dose, it is essential to understand where an individual stays in a contaminated area and for how long.

Y. Tao $(\triangle) \cdot$ M. Kanno

Approved Specified Non-Profit Corporation for Resurrection of Fukushima,

Fukushima, Japan

e-mail: taoyoichi@bridge.ocn.ne.jp

S. Obara $\cdot$ S. Kuriyama $\cdot$ T. Sano $\cdot$ K. Ninomiya

Approved Specified Non-Profit Corporation for Resurrection of Fukushima, Tokyo, Japan 


\subsection{Introduction}

Iitate Village is located 30-50 km northwest of Fukushima Daiichi nuclear power plant. The entire village was designated as deliberate evacuation area in April 2011. On March 31st, 2017, the evacuation order was lifted with the exception of the Nagadoro district. Figure 14.1 shows where the evacuation orders (dotted pattern) have been lifted in March and April 2017 (gray areas are still under evacuation orders).

Prior to the survey in March-June 2017, we have been conducting monthly regional ambient radiation dose measurements in Iitate Village for 6 years. We used two cars equipped with a five/three-inch NaI scintillation survey meters, and GeigerMueller counters connected to GPS, and have displayed the results on maps (e.g.,

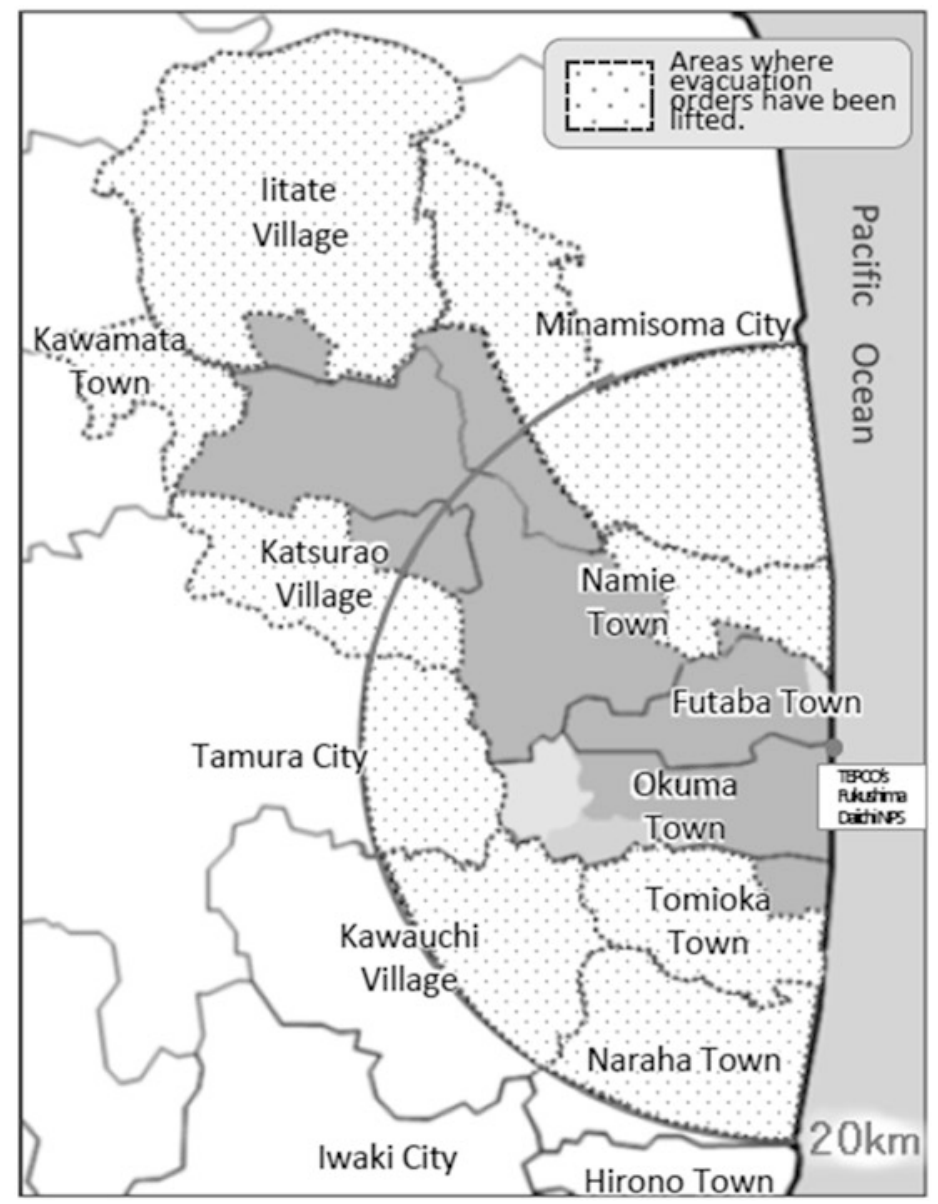

Fig. 14.1 The location and status of Iitate Village. (Source: "Fukushima Revitalization Station" http://www.pref.fukushima.lg.jp/site/portal-english/) 


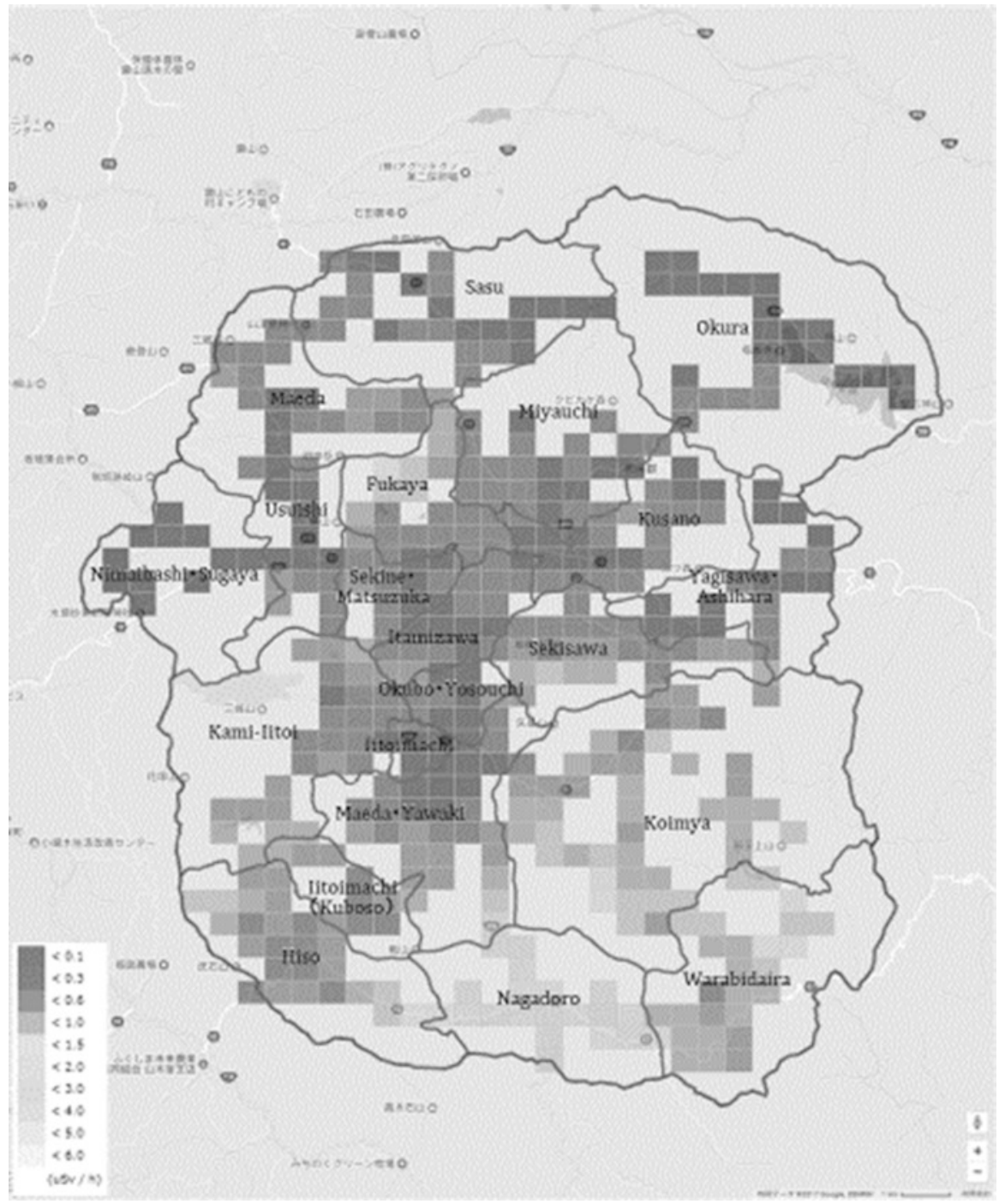

Fig. 14.2 Radiation dose rate in Iitate village from October to December 2016

Fig. 14.2). Forty evacuees from Iitate Village have regularly participated in the radiation measurement. Ambient radiation dose in the northern region is relatively lower than that in the southern region such as Nagadoro, Hiso and Warabidaira. Figure 14.3 depicts attenuation of ambient radiation due to the passage of time, which is faster than the theoretical attenuation of radiation. We also installed 20 fixed units in mountainous areas as well as in private homes in the village to measure ambient dose and conducted a 24-h continuous measurement. We have made precise measurements of participants out walking in the forests and fields. From these various 
1.2

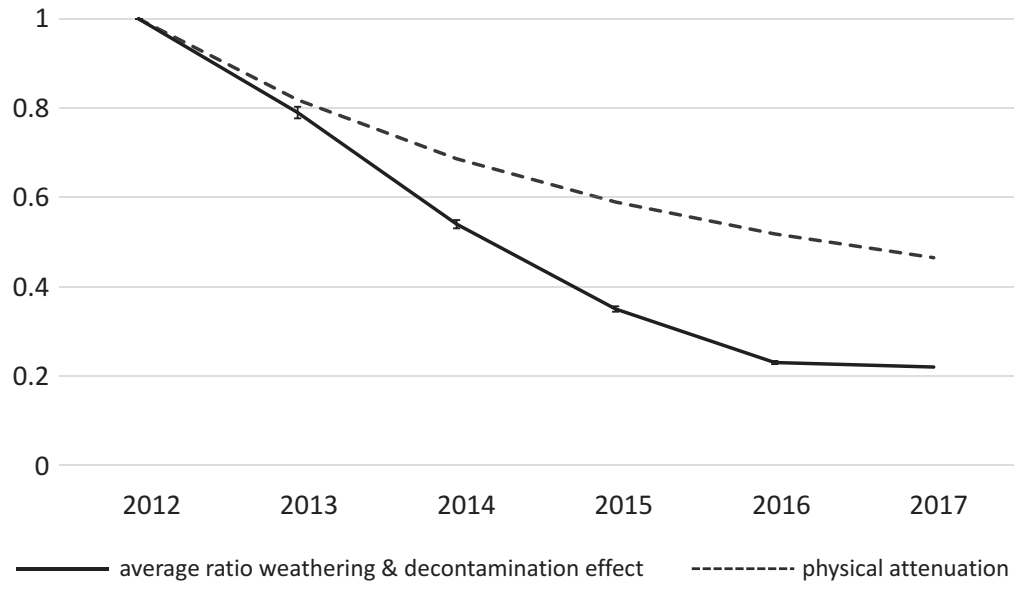

Fig. 14.3 Relative air radiation dose rate from 2012 to 2017 in Iitake village

measurements, we have accumulated ambient dose data for Iitate Village (Resurrection of Fukushima, NPO, Resurrection of Life and Industry in Fukushima, Empathy and Collaboration 2015; Resurrection of Fukushima, NPO, Monitoring Jouhou (Information) 2012-2017). Our purpose is to provide residents with accurate radioactivity data so that they will have the information necessary to decide if they should move back to the village permanently or move elsewhere to rebuild their lives.

\subsection{Methods}

Parallel Measurement of Ambient and Individual External Radiation is based on a survey that we conducted March-June 2017. Two evacuees from Iitate Village and four members of our NPO took part. All participants worked in the area each day. Each participant carried an ambient radiation measuring device, two types of personal radiation measuring devices, and a GPS (Global Positioning System) receiver, allowing us to record their movement and to compare ambient and individual external doses. To ensure the accuracy of ambient dose, participants spent some time beside fixed radiation monitoring equipment that the central government, Fukushima Prefectural Office and Iitate Village authorities installed at various locations throughout the village to assure cross-calibration.

We have utilized the following instruments: NaI scintillation survey meters, ALOKA TCS-172; electronic pocket dosimeter PDM-501 (Hitachi Healthcare) and electronic personal D-Shuttle dosimeter (Chiyoda Technol Corporation, Joint 
development with the National Institute of Advanced Industrial Science and Technology (AIST). By connecting the recording devices to a TCS-172, we recorded the dose every second and then calculated the average hourly dose. All participants carried the TCS-172 in their hand, at a height of about $1 \mathrm{~m}$ above the ground while moving on foot. While working in the field or on the farm, the measurement devices were placed on a table or tripod, not directly on the ground. While driving, the devices were put on the passenger seat or on the luggage rack. For a comparison of dosimeter measurements, all participants wore both a PDM-501 and a D-Shuttle. The PDM-501 was set to record the dose every $5 \mathrm{~min}$. The average hourly dose was then calculated. The dose on the D-shuttle was calculated every hour. In this report, we explain three cases. Figure 14.4 shows each participant's monitoring route.

\subsection{Results and Discussion}

Figure 14.5 (Participant A), Fig. 14.6 (Participant B) and Fig. 14.7 (Participant A) show the overall results of the daily measurements of Participants A and B. The participants stayed in Iitate Village during the day and slept not far away in Ryozen, Ishida District, Date City, where the dose was lower than that in Iitate. The dose in the southern part of Iitate, such as in Nagadoro, Hiso, and Komiya, was higher than that in the northern areas like Sasu and Matsuzuka

Figure 14.5 depicts the results of measurements taken from 10:00 a.m. on April 15 to 12:00 p.m. on April 16, 2017. During this period, the individual started outdoor activities at 10:00 a.m. in Hiso, then went to Sasu around 12:00 p.m. and worked in the field there until 6:00 p.m. He left the measurement devices in the Sasu office until 5:00 a.m. the following morning; the next day, the 16th, he was in Ishida, Date City, working inside and out from 5 a.m. to 8 a.m. He then went back to Sasu and worked in the field there until 12:00 p.m. The solid line shows the ambient dose measured on the TCS-172. The dotted line in the figure shows $70 \%$ of the ambient dose. It approximately matches the gray bars, representing the measurement of the pocket dosimeter PDM-501. Measurements from the D-Shuttle dosimeter are shown as a dark blue bar in the chart. The D-Shuttle measurements tended to be somewhat higher than those of the PDM-501 and of the dispersion of the readings.

Figure 14.6 shows the results of measurements from 1:00 p.m. on June 17 to 3:00 p.m. on June 18th, 2017. This individual left Sasu by car around 1:00 p.m., drove to Hiso and Nagadoro in the southern part of Iitate Village, then went on to Itamisawa and Matsuzuka in the central part of the village. He spent approximately 30 min outside the car in each place. He stayed in a lodging house in Ishida, Date City (not far from litate Village) from 5:00 p.m. to 8:00 a.m. the following morning. He worked in the field in Sasu from 8:00 a.m. to 12:00 p.m., then had lunch in the Sasu office. After lunch, he went to Komiya and returned to Sasu. We found that, as on the previous day, the D-shuttle calibration was higher than that of the DPM501, which was approximately $70 \%$ of the ambient dose. 


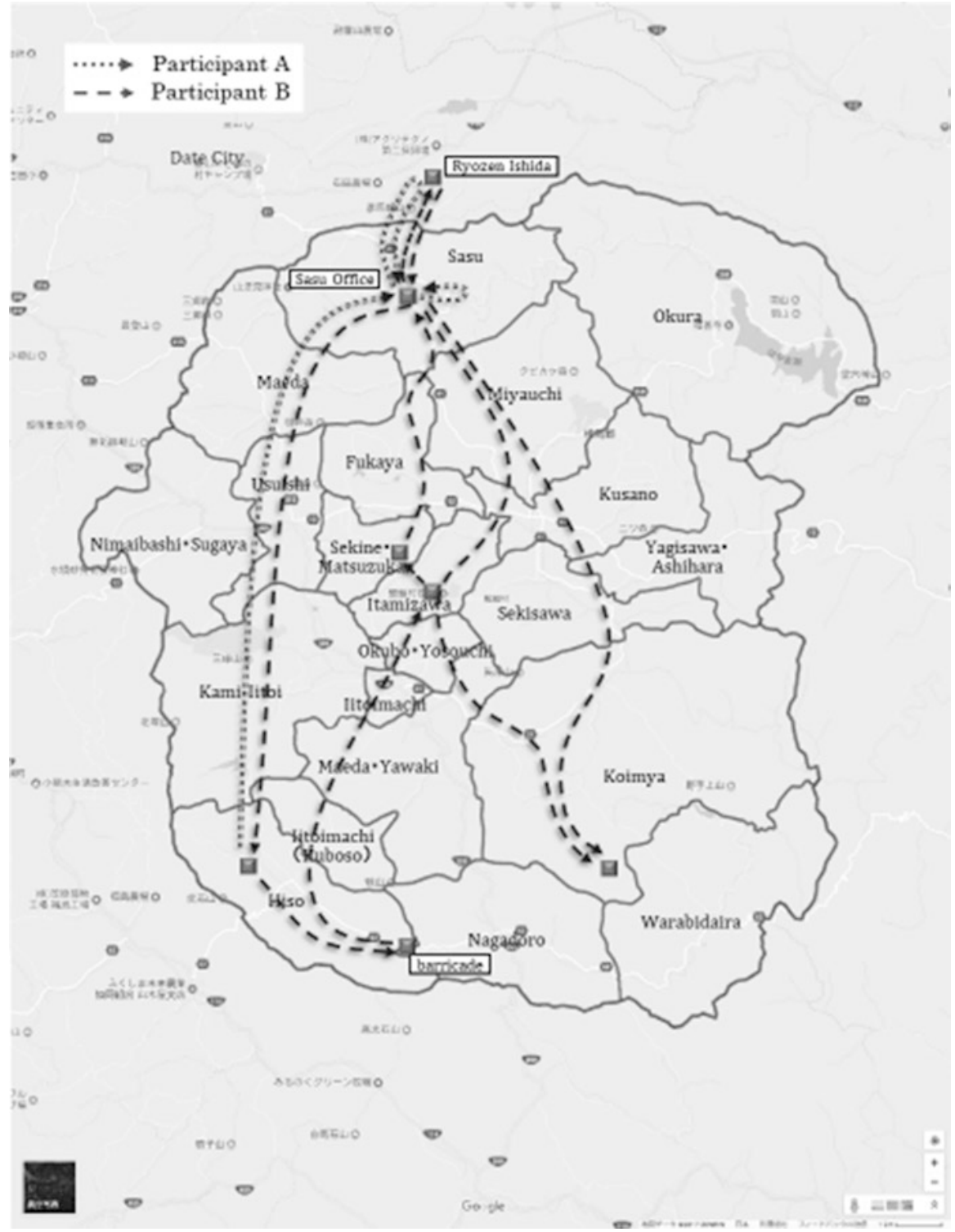

Fig. 14.4 Participant A's monitoring route on April 15th-16th, 2017 and participant B's route on June 17th-18th, 2017

Figure 14.7 indicates the results of measurements taken from 9:00 a.m. to 1:00 p.m. on September 3rd, 2017. This individual started outdoor activities at 9:00 a.m. in Nagadoro and stayed in a community house around 11:00 p.m. to noon, then went to Sasu. 


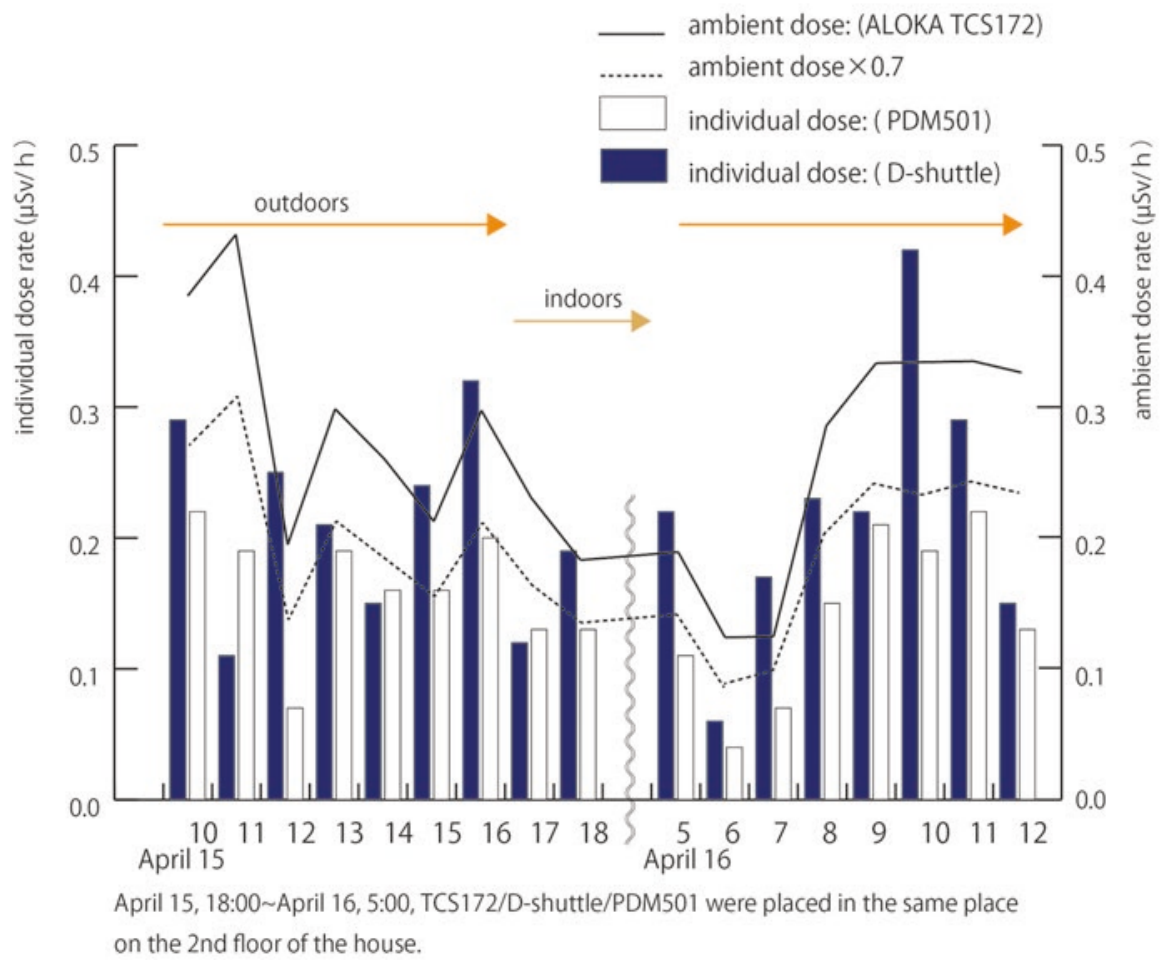

Fig. 14.5 Relationship between individual (Participant A) dose and ambient dose. Monitoring occurred on April 15th-16th, 2017

The results for those who took the same route for the same amount of time showed that regardless of where the person was, the individual dose was about $11 \%$ 30\% lower than that of the ambient dose; the ratio of ambient to individual external dose was 100 to 70-89. The individual integral dose obviously depended on the lifestyle pattern and location of each individual.

Measurement data made available to the public by the central government and affiliated organizations are primarily the radiation ambient dose (per hour) obtained from airborne monitoring and from fixed monitoring posts. Airborne monitoring data is valuable to understand the average ambient dose within a radius of $300 \mathrm{~m}$ (Nuclear Regulation Authority 2017). This data was useful for policymaking as well as measuring radiation levels in highly contaminated areas right after the Fukushima Daiichi nuclear power plant accident (Yuuki et al. 2014).

We found a high correlation between the individual dose and ambient dose simultaneously measured in the same location. Needless to say, the ambient dose was very different at each point, whether in a house, forest, field or on the street, even in places located within a $300 \mathrm{~m}$ radius. When we study individual dose in a 
Participant B: June 17 18, 2017 Relationship between Individual Dose and Ambient Dose
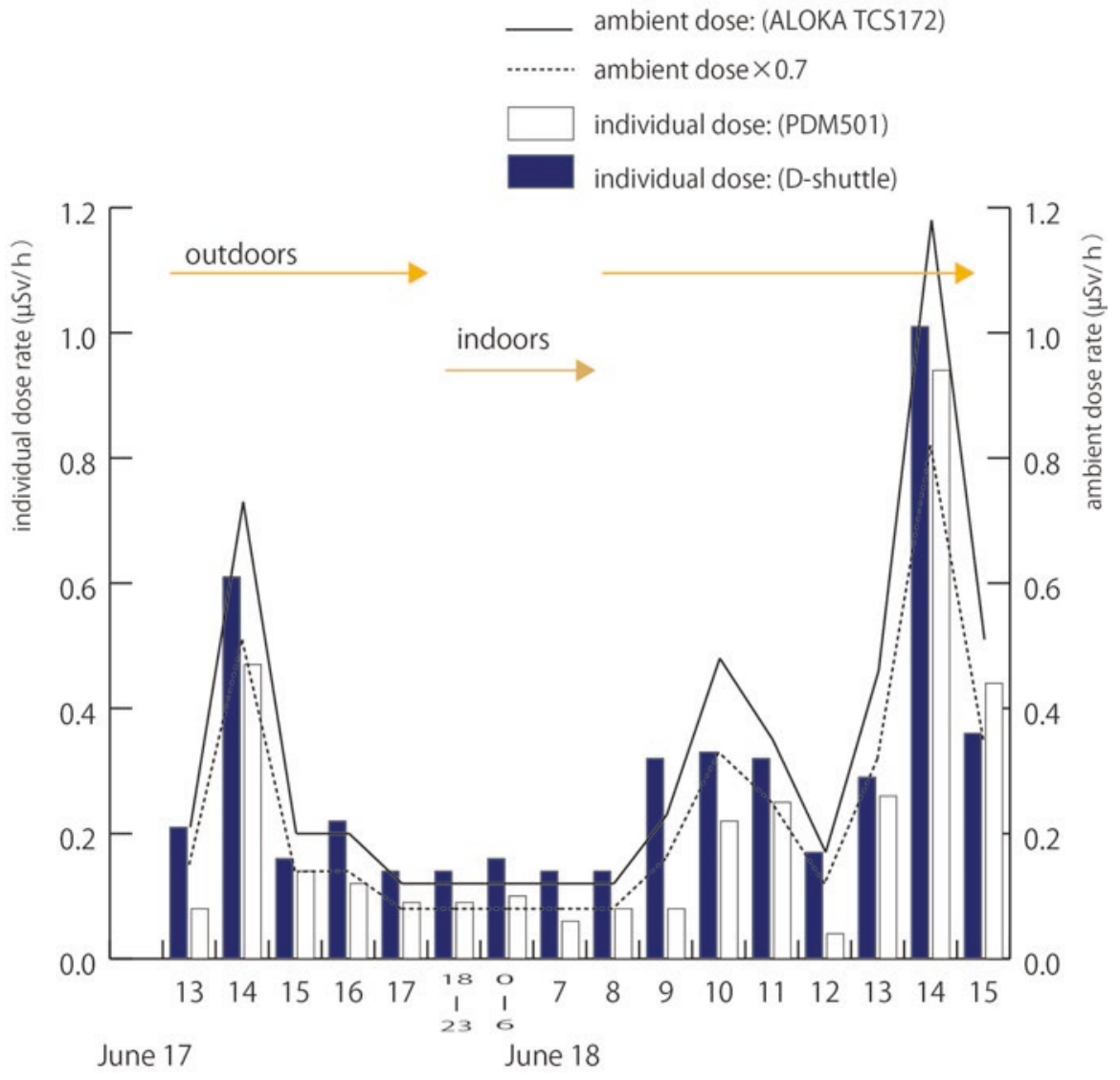

June $17,17: 00 \sim$ June $18,8: 00$, the ambient dose used was $0.12 \mu \mathrm{Sv} / \mathrm{h}$ measured at the same place.

Fig. 14.6 Relationship between individual (Participant B) dose and Ambient Dose. Monitoring occurred on June 17th-18th, 2017

contaminated area, it is essential to be aware of the individual's location, how long he or she stays there and the ambient dose of radiation at the site. A comparison between ambient dose and individual dose without consideration of those elements may cause misunderstanding.

Some studies have reported the relationship between ambient dose obtained from airborne monitoring and individual dose (Ishikawa et al. 2016; Naito et al. 2017; Miyazaki and Hayano 2017a, b). We doubt the relevancy of using airborne monitoring data in relation to individual dose, as was done, for example, for the Date City's glass badge monitoring (Miyazaki and Hayano 2017a, b). This combination cannot 


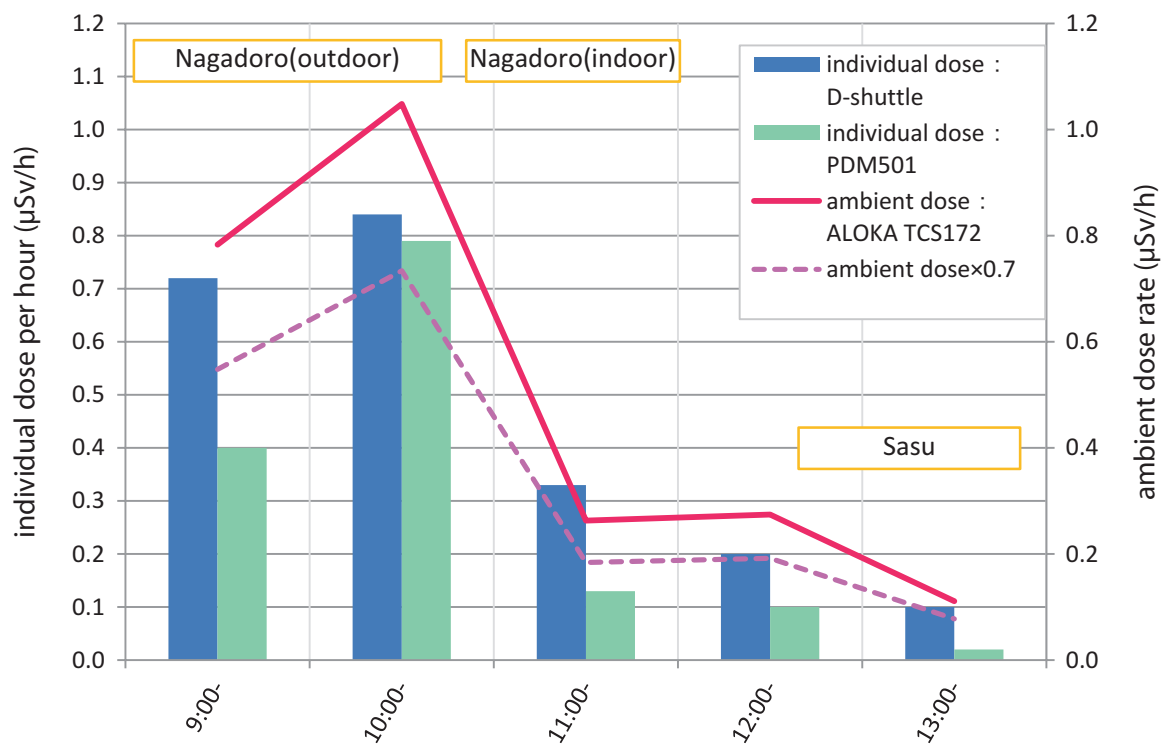

Fig. 14.7 Relationship between individual (Participant A) dose and ambient dose. Monitoring occurred on September 3rd, 2017

be scientifically analyzed with reliable results. Researchers must consider two points when measuring individual external dose: (1) to record the exposure to each individual by location/length of time and (2) to consider only individual data, not the mean. Recent studies ignoring these two points have caused misunderstanding of the current situation in Fukushima (Kornei 2017). Besides, we would like to point out that airborne monitoring data are usually higher than ambient dose monitoring on the ground such as fixed monitoring posts. The airborne monitoring data is an estimated value from $1 \mathrm{~m}$ above the ground. The difference between airborne monitoring and ground monitoring from 2013 to 2016 will be larger in Iitate Village by our estimate. Many Date city residents who received glass badges did not wear them outdoors and simply sent them back to the local government office every 3 months after receiving replacements (The minutes of the proceedings of Date council 2016; Date City council News 2017). In other words, it is not known how many of those glass badges were never worn outside during those 3 months. A comparison between individual dose and ambient dose in a rather wide area without accurate information on location and length of time as well as a system to maintain individual dose records is not useful for understanding the current situation in Fukushima. Even worse, it can mislead residents in Fukushima into thinking that the exposure dose is lower than it actually is. We must offer proper scientific facts to prevent misunderstanding. 


\subsection{Conclusion}

When the evacuation order was lifted in April 2017, some evacuees from Iitate Village returned to work there. Some resumed farming, others reopened their businesses, even though there are still quite a few areas where relatively high radioactivity is observed. Not all returnees are staying overnight in Iitate. Individual dose is highly dependent on each person's actual activities, and those activities vary. Although the radiation level in decontaminated areas has gone down in litate Village, the level in the forests and mountains is higher than in the residential areas because they have not been decontaminated. More thorough radiation measurement is needed for the safety of residents and for further analysis.

Acknowledgments We would like to thank the High Energy Accelerator Research Organization (KEK) for providing the high spec measuring equipment and the calibration. We also are grateful to the Iitate Village Office and the evacuated residents for giving us the opportunity to jointly make monthly measurements for more than 5 years. Mr. Tadashi Ogawa, president of Knowledge Design, has visualized our monthly measurements on maps from the very first stage of our project. Professor Masaru Mizoguti, Graduate School of Agricultural and Life Science / the University of Tokyo, cooperated in taking measurements in fields and forests. Ms. Itsuko Yano supported in arranging and recording. The Mitsui \& Co. Environment Fund has given activity grants. We greatly appreciate their contribution. We hope that more related organizations and individuals will become interested in and work on accurate data collection and accumulation.

\section{References}

Date City council News, (Date Shigikai Dayori) vol 45(10) (2017). http://www.city.fukushimadate.lg.jp/uploaded/attachment/28009.pdf. Accessed 20 July 2017

Ishikawa T, Yasumura S, Ohtsuru A, Sakai A, Akahane K, Yonai S, Sakata R, Ozasa K, Hayashi M, Ohira T, Kamiya K, Abe M (2016) An influential factor for external radiation dose estimation for residents after the Fukushima Daiichi Nuclear Power Plant accident-time spent outdoors for residents in Iitate Village. J Radiol Prot 36:255. Accessed 20 July 2017

Kornei K (2017) Fukushima residents exposed to far less radiation than thought. Science. https:// doi.org/10.1126/science.aal0641. Accessed 20 July 2017

Miyazaki M, Hayano R (2017a) Individual external dose monitoring of all citizens of Date City by passive dosimeter 5-51 months after the Fukushima NPP Accident (series): I. Comparison of individual dose with ambient dose rate monitored by aircraft surveys. J Radiol Prot 37(1). IOPscience (2016). Accessed 20 July 2017

Miyazaki M, Hayano R (2017b) Individual external dose monitoring of all citizens of Date City by passive dosimeter 5 to 51 months after the Fukushima NPP accident (series): II. Prediction of lifetime additional effective dose and evaluating the effect of decontamination on individual dose. J Radiol Prot 37:623. IOPscience (2017). Accessed 14 Aug 2017

Naito W, Uesaka M, Kurosawa T, Kuroda Y (2017) (. Accessed 20 July 2017) Measuring and assessing individual external doses during the rehabilitation phase in Iitate village after the Fukushima Daiichi nuclear power plant accident. J Radiol Prot 37:606

Nuclear Regulation Authority, Results of the Eleventh Airborne Monitoring and Airborne Monitoring out of the 80km Zone of Fukushima Dai-ichi NPP February 13, 2017. http://radioactivity.nsr.go.jp/en/contents/12000/11830/24/11th\%20Airborne_eng.pdf. Accessed 20 July 2017 
Resurrection of Fukushima, NPO, Monitoring Jouhou (Information) (2012-2017) Monitoring information. http://rad.fukushima-saisei.jp/mesh.html. Accessed 20 Aug 2017

Resurrection of Fukushima, NPO, Resurrection of Life and Industry in Fukushima, Empathy and Collaboration (2015) Resurrection of Fukushima activity report http://www.fukushimasaisei.jp/app-def/S-102/madei/wp-content/uploads/2015/06/Presentation-package-as-ofJune-10-2015.pdf. Accessed 20 Aug 2017

The minutes of the proceedings of Date council, December 8, (2016) 196-198. http://www.kaigiroku.net/kensaku/datecity/datecity.html. Accessed 20 July 2017

Yuuki Y, Maeshima M, Hirata R, Matsui M (2014) Airborne radiation monitoring in the Fukushima Daiichi nuclear power plant accident, Oyo technical report, pp 106-115. Accessed 20 July 2017

Open Access This chapter is licensed under the terms of the Creative Commons Attribution 4.0 International License (http://creativecommons.org/licenses/by/4.0/), which permits use, sharing, adaptation, distribution and reproduction in any medium or format, as long as you give appropriate credit to the original author(s) and the source, provide a link to the Creative Commons license and indicate if changes were made.

The images or other third party material in this chapter are included in the chapter's Creative Commons license, unless indicated otherwise in a credit line to the material. If material is not included in the chapter's Creative Commons license and your intended use is not permitted by statutory regulation or exceeds the permitted use, you will need to obtain permission directly from the copyright holder. 